\title{
Real-time ultrasound guided technique for improving the safety of Internal Jugular vein cannulation
}

\author{
Authors \\ Kishore K Baliga ${ }^{1}$, Geetha N.K. ${ }^{2 *}$, Anil Sathyadas ${ }^{3}$
}

${ }^{1}$ Junior Resident, Department of Anaesthesiology, Government Medical College, Thiruvananthapuram, Kerala ${ }^{2}$ Additional Professor, Department of Anaesthesiology, Government Medical College, Thiruvananthapuram, Kerala

${ }^{3}$ Associate Professor, Department of Anaesthesiology, Government Medical College, Thiruvananthapuram, Kerala

*Corresponding Author

Geetha N.K.

Additional Professor, Department of Anaesthesiology, Government Medical College, Thiruvananthapuram, Kerala Ph: 9447745863; email: nkdrgeetha@gmail.com

\begin{abstract}
Internal Jugular Vein is routinely cannulated for long term infusion of drugs irritating the peripheral veins, vasopressor therapy, total parenteral nutrition, chemotherapy agents, antibiotics, repeated blood sampling, trans venous cardiac pacing and haemodialysis in critical care patients. The safe puncture of internal jugular vein can be achieved by using anatomical landmarks in the neck region. However, complications like carotid artery puncture, haematoma, haemothorax, pneumothorax, catheter malposition or even death can occur as consequences of the procedure. Ultrasound guidance could be beneficial in reducing the number of needle passes and decreasing the complications. The present study observed the cannulation techniques of internal jugular vein by anatomical landmark guided and real-time ultrasound guided approach and compared whether the use of ultrasound improves the safety of the technique. In this study, hundred patients requiring right internal jugular vein cannulation via central approach were allocated to one of the two groups- anatomical landmark guided or real-time ultrasound guided group (50 in each group) based on the technique of cannulation. In our study, there was $86 \%$ success rate for first attempt cannulation in the ultrasound guided technique and 68\% in the anatomical landmark guided technique. The complication rates were 7 (14\%) in ultrasound guided group vs. $19(38 \%)$ in the landmark guided group. Ultrasound guided technique improves the catheterization technique of the internal jugular vein with respect to safety of the procedure.
\end{abstract}

Keywords: Central venous cannulation, Internal Jugular Vein, Anatomical Landmark, Real-time ultrasound.

\section{Introduction}

Central vein cannulation plays an important role during resuscitation of patients in critical care as well as emergency room. The central vein cannulation provides a long term and continuous intravenous route for administering drugs, repeated blood sampling and nutrition. Use of central venous catheterization and cannulation devices has become an important aid in the management of critically ill patients ${ }^{1}$. It has an important role in disease management of surgicaloncological patients and hemodialysis patients ${ }^{2,3}$. 
The filling pressure measurements afforded by monitoring central venous pressure allow differentiation between hypovolemia and myocardial depression ${ }^{4,5}$. Central venous pressure can also correlate with changes in pulmonary and left ventricular end diastolic pressure, thereby reflecting left ventricular function ${ }^{6}$. The introduction of these central venous cannulation techniques has allowed the optimal adjustment of circulatory variables by direct haemodynamic measurements and volume replacement, infusion of a wide variety of pharmacologically active agents which cannot be satisfactorily infused via peripheral veins and provision of intravenous nutrition for prolonged periods of time ${ }^{2}$.

Percutaneous right internal jugular cannulation is favoured for various reasons ${ }^{7}$. Various positions were used to access cannulation but they were frequently associated with immediate mechanical complications and respiratory compromise such as arterial \& venous punctures, cardiac tamponade, airway compression by haematoma, haemo/ pneumothorax as well as neurological damage and dysrhythmias. Delayed complications like clogging of the catheter, thromboembolic and infectious complications were also associated with it ${ }^{8}$.

Real time ultrasonography is a useful tool to study the variations in the size of IJV according to various body positions; in finding out the maneuvers which maximize the IJV diameter and in localizing the site of percutaneous puncture. It thereby helps in increasing the first pass success rate and improves the quality of technique by adding safety and lessening the complications.

Hence, this prospective observational study tried to analyze the real-time ultrasound guided technique and the classical anatomical landmark technique of right IJV cannulation (central approach) in terms of number of skin punctures and the immediate complications related to the technique that has occurred in both the groups.

\section{Materials and Methods}

Institutional Research and Ethical Committee clearances were obtained. Hundred patients, above
18 years of age of either sex, admitted in the Critical Care Unit, requiring central venous cannulation for various indications and who gave their informed written consent, were the source of the study.

Patients with traumatic injury/obstruction to superior vena cava, in nominate or internal jugular vein; severe bleeding tendencies and coagulopathy states; fungating tricuspid valve vegetation; severe respiratory distress, tachypnea, \& labored breathing; restless and uncooperative patients and patients with refusal to take part in the study or retraction of previously given consent were excluded from the study.

A prospective, observational study, with a prior calculated sample size of 50 cases per group, was conducted. The group of patients in whom realtime ultrasound guided technique was used was termed the ultrasound guided group (USG group) whereas the patients in whom anatomical landmark technique was used was labelled the anatomical landmark guided group (LMG group). The above two groups were observed for their right IJV cannulation techniques (central approach).

Real-time ultrasound guided as well as anatomical landmark guided techniques were followed for IJV catheterization as per the discretion and expertise of the consultant intensivist and the availability of the ultrasound machine in the CCU. All consecutive patients eligible for the study as per inclusion and exclusion criteria and requiring right IJV cannulation (central approach) were enrolled into either the 'USG group' or the 'LMG group' based on the technique of cannulation and observed till required sample size was achieved.

Detailed clinical history, physical examination and written informed consent of the patient was taken along with age, sex, BMI and baseline recordings of heart rate, ECG, blood pressure and O2 saturation. Routine blood and urine investigations, coagulation profile (platelet count, bleeding time, clotting time, PT-INR, APTT) as well as Chest X-ray (CXR) findings were also recorded. A peripheral IV access, resuscitation 
equipments and drugs were kept ready before starting the procedure. The Seldinger technique was employed for cannulation.

Patient was kept in Trendelenburg (15-20 $)$ position with head turned slightly towards the left side and stabilized with folded towels. After assessing the anatomical landmarks (sternocleidomastoid muscle-SCM, sternal notch, cricoid cartilage, clavicle and carotid artery pulsations) right side of the neck was prepared with an antiseptic solution. The procedure was carried out under deep sedation and local infiltration with local anesthetic solution.

In the real-time ultrasound guided technique, the ultrasound probe, covered in a sterile sheath, was placed over the triangle formed by the two heads of the sternocleidomastoid muscle (SCM) and the vessels were visualized in the transverse section. The seeker needle was then inserted under visualization on the US screen. A J-tipped guide wire was inserted through the hollow needle after successful aspiration of venous blood and after single passage of a dilator through the guide wire. A triple lumen central venous catheter was then rail-roaded over the guide wire. All ports of the central line were checked for free flow of blood after the guide wire was withdrawn. After suturing the catheter to the skin, a transparent dressing was applied over the area of insertion.

For the anatomical landmark guided technique, the apex of the triangle formed by the two SCMs was palpated for internal carotid artery (ICA) pulsations. The ICA was then pressed slightly medially with the left hand so that it did not overly the IJV. A $22 \mathrm{G}$ needle attached to $5 \mathrm{ml}$ syringe containing heparinized saline was advanced through the skin just lateral to the point of ICA pulsation at an angle of $20^{\circ}-30^{\circ}$ to the skin and directed towards the ipsilateral nipple. A $18 \mathrm{G}$ needle connected to a $5 \mathrm{ml}$ syringe, the seeker needle, was inserted after aspirating blood venous blood with the finder needle and using it as a guide. After successful aspiration of blood with the seeker needle, rest of the steps were similar to that in ultrasound guided technique.
Following observations were made:

- Number of attempts taken to access the IJV. Success rate: $-\leq 3$ attempts to access the vein was termed successful access.

- Any immediate complications namely carotid artery puncture, arrhythmias, skin hematoma, nerve injuries, catheter misplacement and haemo/pneumothorax.

- Position of tip of the catheter: - assessed by checking for back flow, palpating the ICA medial to the puncture site, measuring baseline CVP and CXR-PA view after the procedure

Data was analyzed using Statistical Package for the Social Science software trial version 18 and Microsoft Excel. Quantitative data was analyzed by computing percentages and descriptive statistics viz., mean, standard deviation, and standard error of mean and qualitative data by means of proportions. Quantitative data between the two groups was compared using the Independent sample t-test and qualitative data by means of Chi-square test. Results were considered statistically significant whenever $\mathrm{p} \leq 0.05$.

\section{Observation and Analysis}

The demographic profile, with respect to age, sex and BMI, were comparable between the groups (Table 1) and the differences were statistically insignificant. ( $p>0.05)$.

Out of the 100 patients selected for the study, 77 patients were cannulated in the first attempt and 18 in the second attempt. 4 patients required three attempts for cannulation and only a single patient required four skin punctures for catheterization. Using LMG technique $34(68 \%)$ cases were cannulated in first attempt, followed by $11(22 \%)$ cases in second attempt and $4(8 \%)$ cases in the third attempt. 1 case (2\%) required 4 skin punctures for cannulation. However, in the USG group, $43(86 \%)$ cases were cannulated in the first attempt itself and $7(14 \%)$ cases required 2 nd attempt for cannulation. Unlike the LMG group, no cases needed 3rd or 4th attempt. Therefore, USG technique clearly reduces the number of 
attempts for cannulation (Figure 1). However, in this study, the observed variable i.e. cannulation attempt, was not statistically significant [Chisquare value-6.941, Degree of freedom- 3 and pvalue -0.074].

In this study, Carotid artery puncture accidently occurred in $4(8 \%)$ cases in the LMG technique where as in USG technique there was no case of carotid artery puncture. p- value was 0.041 and was statistically significant (Table 3 ).

A total of 12 patients developed hematoma during the cannulation procedure ( 8 cases in the LMG group and 4 cases in the USG group). The difference observed was not statistically significant $(\mathrm{p}=0.218)$ (Table 3$)$.

7 patients developed arrhythmias during cannulation using the LMG technique and 3 patients using the USG technique. However, the results obtained were not statistically significant $(\mathrm{p}=0.182)$ (Table 3$)$.

In both the groups, there were no cases of pneumothorax, haemothorax or nerve injuries following cannulation.

Table 2 and Figure 2 shows the distribution of complications encountered during the study.
Guide wire related problems (difficulty in threading the wire) were noted in 6 patients cannulated using LMG technique and 3 patients using USG technique. Catheter related problems (difficulty in threading the catheter) occurred in 5 patients of the LMG group and 2 patients of the USG group. But both these variables were statistically not significant (p-value of 0.295 and 0.240 respectively (Table 4$)$.

The position of the tip of the catheter following IJV cannulation was confirmed in all cases of both the groups using Chest $\mathrm{X}$-ray postero-anterior (PA) view. 3 cases in LMG group and 1 case in USG group needed repositioning because of abnormal position of the catheter tip (Figure 3).

Table 1: Demographic distribution among study groups

\begin{tabular}{|c|c|c|c|}
\hline Variables & LMG $(n=50)$ & USG $(n=50)$ & p value \\
\hline $\begin{array}{l}\text { Age } \\
\text { (years) }\end{array}$ & $50.32 \pm 15.61$ & $49.46 \pm 16.15$ & $p-0.787$ \\
\hline $\begin{array}{l}\text { BMI } \\
\left(\mathrm{kg} / \mathrm{m}^{2}\right)\end{array}$ & $23.71 \pm 2.68$ & $23.25 \pm 3.30$ & $p-0.452$ \\
\hline $\mathrm{Sex}^{*}$ & $\begin{array}{ll}\text { Male } & 29(58 \%) \\
\text { Female } & 21(42 \%) \\
\end{array}$ & $\begin{array}{ll}\text { Male } & 27(54 \%) \\
\text { Female } & 23(46 \%) \\
\end{array}$ & $\mathrm{p}-0.687$ \\
\hline
\end{tabular}

LMG - Landmark guided group; USG - Ultrasound guided group; BMI - body mass index

Data as mean \pm standard deviation; student's t-test

*Data as number of patients (percentage); Chi square test $\mathrm{p}$ value $<0.05$ is considered statistically significant

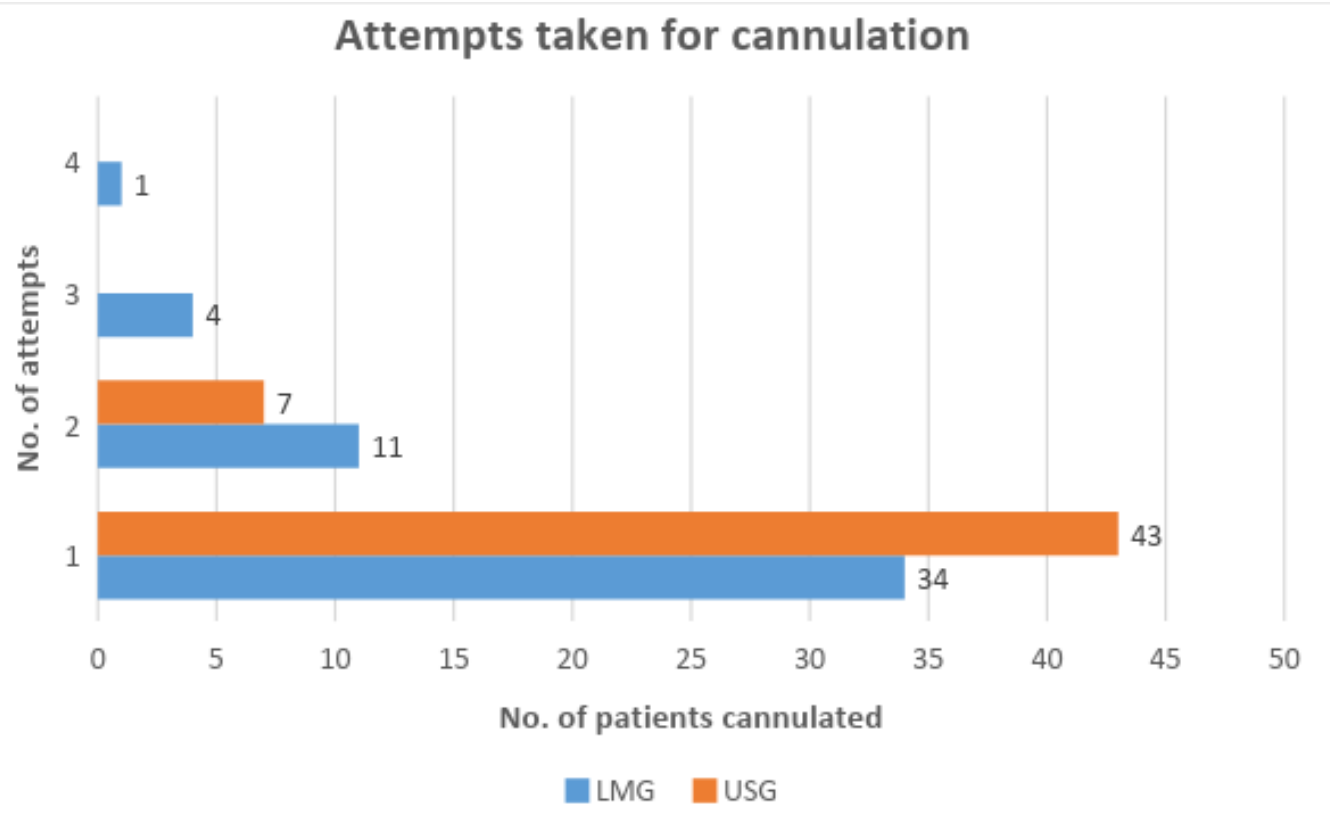

Figure 1: Distribution of cannulation attempts among the study groups.

LMG - Landmark guided group; USG - Ultrasound guided group 
Table 2: Immediate complications noted among the study groups

\begin{tabular}{|l|c|c|c|c|c|c|}
\hline Complications & $\begin{array}{c}\text { CA } \\
\text { puncture }\end{array}$ & Haematoma & Arrhythmia & $\begin{array}{c}\text { Pneumo- } \\
\text { thorax }\end{array}$ & $\begin{array}{c}\text { Haemo- } \\
\text { thorax }\end{array}$ & Nerve injury \\
\hline LMG(n=50) & 4 & 8 & 7 & 0 & 0 & 0 \\
\hline USG(n=50) & 0 & 4 & 3 & 0 & 0 & 0 \\
\hline TOTAL(n=100) & 4 & 12 & 10 & 0 & 0 & 0 \\
\hline
\end{tabular}

LMG - Landmark guided group; USG - Ultrasound guided group; CA - Carotid artery

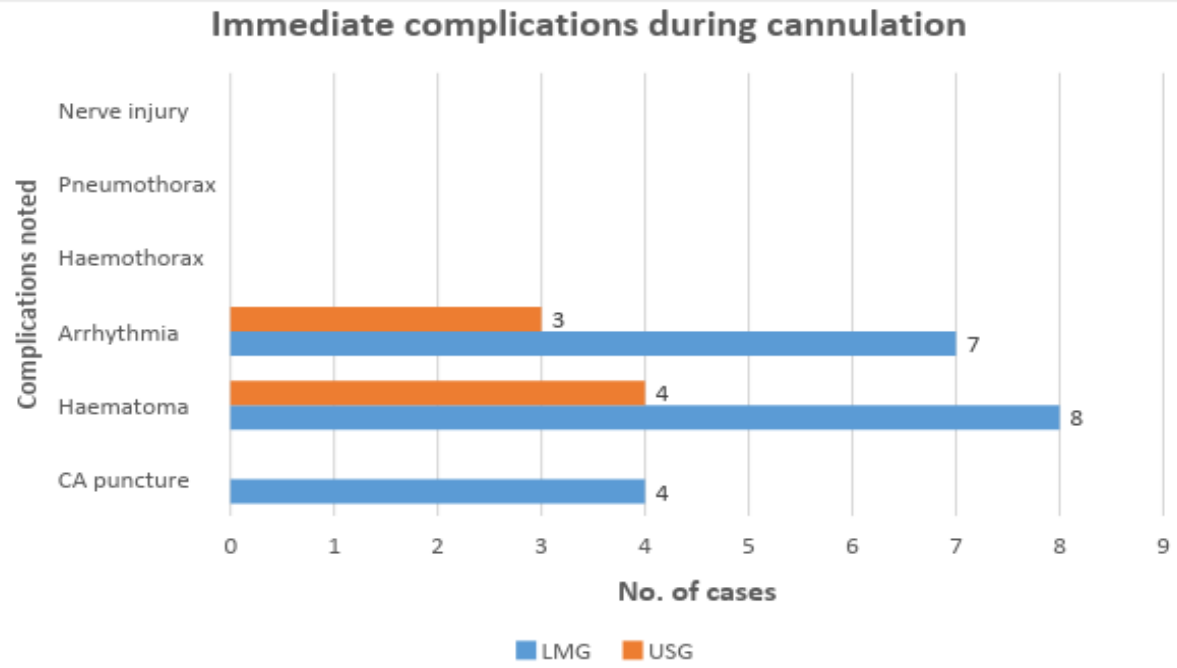

Figure 2: Immediate complications noted among the study groups LMG - Landmark guided group; USG - Ultrasound guided group; CA - Carotid artery

Table 3: Analysis of various statistical variables among the study groups

\begin{tabular}{|l|c|c|c|}
\hline Statistical variables & Chi-Square value & Df & 'p' value \\
\hline CA puncture & 4.167 & 1 & .041 \\
\hline Haematoma & 1.515 & 1 & .218 \\
\hline Arrhythmia & 1.778 & 1 & .182 \\
\hline
\end{tabular}

Df - Degree of freedom; CA - Carotid artery $\mathrm{p}$ value $<0.05$ is considered statistically significant

Table 4: Technical issues during cannulation

\begin{tabular}{|c|c|c|c|}
\hline Method & Guide wire issue & Catheter issue & p value \\
\hline LMG $(n=50)$ & 6 & 5 & $\mathrm{p}-0.295$ \\
\hline USG $(\mathrm{n}=50)$ & 3 & 2 & $\mathrm{p}-0.240$ \\
\hline
\end{tabular}

LMG - Landmark guided group; USG - Ultrasound guided group; CA - Carotid artery $\mathrm{p}$ value $<0.05$ is considered statistically significant

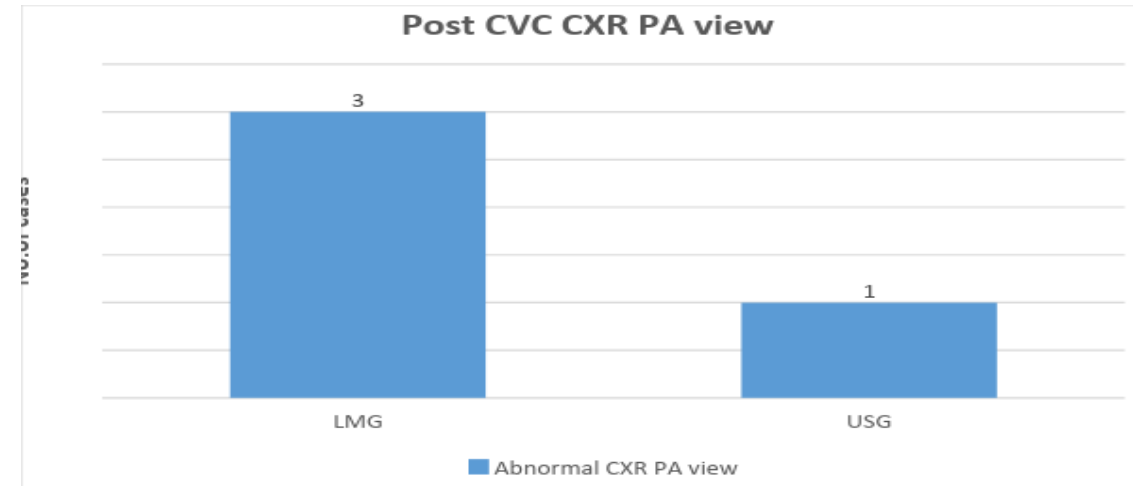

Figure 3: Bar graph showing frequency of abnormal post CVC CXR PA view among the study groups LMG - Landmark guided group; USG - Ultrasound guided group; CVC- central venous cannulation; CXR- Chest X-ray; PApostero-anterior 


\section{Discussion}

The main objective of this study was to find out whether USG guidance reduces the number of skin punctures and improves the safety of right sided IJV catheterization. Various factors including anthropometric parameters were taken into consideration, but parameters like age, gender, height, weight or BMI had no significance in this study.

In short and thick neck patients, there was difficulty in appreciating the anatomical landmarks and so the number of attempts were more in these cases, especially in the LMG group. Using the USG technique number of IJVs entered on the first attempt were considerably higher. In the present study, by using USG technique 43(86\%) patients were cannulated on the first attempt vs $34(68 \%)$ patients by the LMG technique. The results obtained in the present study were comparable to the results obtained by the studies of Dimitrios Karakisos et $\mathrm{al}^{9} 100 \%$ vs $94.4 \%$, Piero Antonio et $\mathrm{al}^{10} 100 \%$ vs $91.6 \%, \mathrm{Wg}$ Cdr R M Sharma et al ${ }^{11} 100 \%$ vs $98 \%$, Testa A et $\mathrm{al}^{12} 100 \%$ vs $82 \%$, Bart G Denys et $\mathrm{al}^{13} 78 \%$ vs $43.3 \%$, Mallory et $\mathrm{al}^{14}$ with $85 \%$ vs $15 \%$ respectively. A maximum of 4 attempts were made in LMG technique $(1.44 \pm 0.73)$ compared with a maximum of only 2 attempts in the USG group $(1.14 \pm 0.35)$ with similar results seen with Testa A.et $\mathrm{al}^{12}{ }^{2} 2.3 \pm 1.3$ vs $1.2 \pm 0.4$, Daniel Duque et al ${ }^{15} 5$ vs 2.3 and Hasan Dolu et al ${ }^{16} 1.1 \pm$ 0.5 vs $2.2 \pm 1.6$. But the number of attempts made to cannulate the vein was not statistically significant as per the present study.

There was a significant decrease in complication with use of ultrasound-guided technique. The complication rates were 19 in the LMG group vs. 7 in the USG group. The carotid artery was punctured in 4 patients in the LMG group compared with no cases in the USG group comparable with Dimitrios Karakisos et $\mathrm{al}^{9} 10.6 \%$ vs $0 \%$, Piero Antonio et $a^{10} 7.7 \%$ vs $0 \%$, Daniel Duque et $\mathrm{al}^{15} 20 \%$ vs $0 \%$, and Gurkan Turker et $\mathrm{al}^{17} 4.73 \%$ vs $0.5 \%$ respectively. Reports from a retrospective analysis by Yeum et $\mathrm{al}^{18}$ in 150 patients who required IJV catheterization showed that arterial puncture of the common carotid artery occurred in $11.3 \%$ of the cases. Another study by Shah $\mathrm{KB}$ et $\mathrm{al}^{19}$ reports incidence of carotid artery (CA) puncture ranging from $5.7 \%$ to $13.3 \%$ with anatomical landmark guidance in IJV cannulation. 12 cases of skin hematoma occurred during the study ( 8 in LMG group and 4 in the USG group) when compared with the findings of Dimitrios Karakisos et $\mathrm{al}^{9} 8.4 \%$ vs $0 \%$, Bart G Denys et al ${ }^{13}$ $2.66 \%$ vs $0 \%$, and Gurkan Turker et $\mathrm{al}^{17} 3.68 \%$ vs $1 \%$ in LMG and USG groups respectively.

The other complications related to cannulation rather than technique was ventricular ectopics on ECG monitor while passing the guide wire which was noted in 7 patients in the LMG group and 3 in the USG group which subsequently reverted back to sinus rhythm on adjusting the depth of insertion. There were no serious complications like pneumothorax or nerve injuries in either group when compared to the occurrence of pneumothorax in $2.4 \%$ vs $0 \%$ in Dimitrios Karakisos et $\mathrm{al}^{9}$ study and Testa $\mathrm{A}$ et $\mathrm{al}^{12}$ study of $5.8 \%$ vs $0 \%$ in LMG technique and USG technique respectively.

All the IJV cannulation cases of both the technique were subjected to chest X-ray posterioanterior view for confirmation of position of the catheter tip. The ideal position of catheter tip is parallel to superior vena cava, just below the inferior border of clavicle, above 2-3 ribs, at the tracheal carina. In this study, 3 cases in the LMG group and 1 case in the USG group had abnormal position of the catheter tip. The catheters were subsequently repositioned and chest radiographs were repeated to confirm the final accepted position of the catheter tip.

Hence, the USG technique greatly improves the safety of the procedure of CVC as evidenced by reduction in the immediate complications as well as the number of skin pricks even though only one variable 'carotid artery puncture' was found to be statistically significant as per this study with a pvalue of 0.041 . 


\section{Conclusion}

On the basis of our study, we draw the conclusion that ultrasound guidance is beneficial in placing central venous catheters when compared to the anatomical land mark guided technique as it reduces the number of skin pricks and improves the safety of the procedure. There is considerable reduction in the immediate complications following cannulation of IJV with USG guidance. There are few and less serious complications during catheterization of internal jugular vein with the USG technique even by inexperienced operators. Moreover, the sonographic guidance of central venous cannulation is a very good tool for demonstration, training and teaching the technique to the trainees.

\section{Source of support: Self}

\section{References}

1. Andrew R. Webb, Marc J. Sharpiro, Meryn Singer: Oxford textbook of Critical Care, Oxford Medical Publications 1990: 1090 - 1094

2. Marano L, Izzo G, Esposito $G$, et al. Peripherally inserted central catheter tip position: a novel empiricalultrasonographical index in a modern surgical oncology department. Ann Surg Oncol. 2014; 21: 656-661

3. Duffy BJ J. The clinical use of polyethylene tubing for intravenous therapy; A report on 72 cases.Ann.Surg.1949; 130(5):929-936

4. William R Jernigan, William C Gardner, Merle M Mahr, Joseph L Milbum. Use of Internal Jugular Vein for placement of Central Venous Catheter. Surg, Gyne \& Obst March 1970: 520-524

5. Pat O Daily MD, Randall B Griep MD, Shumway N E. Percutaneous Internal Jugular Vein Cannulation. Arch Surg Oct 1970; Vol. 101: 1103 - 1108

6. Mehmet Tugrul, Emre Camei, Kamil Pemberi, Ayman Al-Darsani, Lufti Telei
U. Relationship between Peripheral and Central Venous Pressure in different patient positions, catheter sizes and insertion sites. $\mathbf{J}$ Cardiothoracic and Vascular Anaes 2004; 18(4): 446 - 450

7. Sally E Mitchell, Robert A, Clark: Complications of Central Venous Catheterization: Am J Roent, September 1979; 133: 467-476

8. Ronald D Miller. Miller's Anaesthesia; $7^{\text {th }}$ Edition; Churchill Livingstone, Elsevier, 2009; 1285 - 1285b: 2586, 2657

9. Dimitrios Karakisos, Nicolas Labropoulos, Eric De Groot, Alexandros P P, Gregorios Kauraksis, John Poulasas et al. Real time Ultrasound guided catheterization of Internal Jugular vein - A prospective comparison with the land mark technique in critical care patients. J Critical Care 2007; Vol 10(6): 843 - 863

10. Piero Antonio Conz, Daniela Dissegna, Maria Pia Rodighero, Giuseppe La Greca. Cannulation of internal Jugular vein Comparison of classical Seldinger technique and ultrasound guided method. J Nephrology 1997; Vol 10(6): 311 - 313

11. Wg Cdr R. M. Sharma, Col C.V.R. Mohan, Lt Col R. Sethur, Wg Cdr H Sahani. Ultrasound guided central venous cannulation. Medical J Armed Forces of India 2006; 62: 371 - 372

12. Testa A., Biasucci D.G., La Greca, Pittiruti M, Portale G, Pignataro $G$, et al. Ultrasound guided central venous cannulation versus the land mark guided technique. J Annals of Emergency Medicine 2004; 11: 582 - 585

13. Bart G Denys, MD, Barry F Uretsky, MD, P Sudhakar Reddy, MD. Ultrasound Assisted Cannulation of the Internal Jugular Vein. Circulation May 1993; 87(5): $1557-1562$

14. Douglas L Mallory, Shawker TH, Evans $\mathrm{RG}$, et al: Effects of clinical maneuvers on sonographically determined internal 
jugular vein size during venous cannulation: Crit Care Med,1990; Vol 18:1269-1273

15. Daniel Duque, Stuart Kessler. Ultrasound guided vascular access. J Intensive Care Medicine 1997; 23: 916 - 919

16. Hasan Dolu, Sttk1 Goksu, Levent Sahin, Onder Ozen, Levent Eken. Comparison of an ultrasound-guided technique versus a landmark-guided technique for internal jugular vein cannulation. Journal of Clinical Monitoring and Computing; February 2015, Volume 29, Issue 1: 177182

17. Turker G, Kaya FN, Gurbet A, Aksu H, Erdogan C, Atlas A. Internal jugular vein cannulation: An ultrasound-guided technique versus a landmark- guided technique. Clinics. 2009; 64(10): 989-992

18. Yeum CH, Kim SW, Nah MY, Ma SK, Ko $\mathrm{JH}, \quad$ Kim $\mathrm{NH}$ et al. Percutaneous catheterization of the internal jugular vein for hemodialysis. Korean J Intern Med. 2001; 16: 242-246

19. Shah KB, Rao TLK, Laughlin S, El-Etr AA. A review of pulmonary artery catheterization in 6,245 patients. Anesthesiology 1984; 61: 271-275. 